\title{
Continuous Electromagnetic Emission Measurement Setup with Antenna Position Tracking
}

\author{
Denys Pokotilov ${ }^{1}$ \\ ${ }^{1}$ University of Twente \\ Enschede, The Netherlands \\ denys.pokotilov@utwente.nl
}

\author{
Robert Vogt-Ardatjew ${ }^{1}$ \\ ${ }^{1}$ University of Twente \\ Enschede, The Netherlands \\ r.a.vogtardatjew@utwente.nl
}

\author{
Tom Hartman \\ ${ }^{1}$ University of Twente \\ Enschede, The Netherlands \\ tom.hartman@utwente.nl
}

\author{
Frank Leferink ${ }^{1,2}$ \\ ${ }^{1}$ University of Twente \\ Enschede, The Netherlands \\ ${ }^{2}$ Thales, Nederland B.V. \\ Hengelo, The Netherlands \\ frank.leferink@utwente.nl
}

\begin{abstract}
For measuring the maximum electromagnetic emissions around equipment many test methods use the frequency domain, traditionally using a super-heterodyne test receiver. However, for large equipment under test the frequency domain method is very time consuming when the measurements are performed at low frequencies, below $150 \mathrm{kHz}$, where small bandwidth and thus long measurement time is needed. Timedomain measurements are now replacing the traditional superheterodyne receivers, but the commercial time-domain receivers still use a first mixer stage. However, at low frequencies, low cost digitisers can do the same job directly in the baseband. Using fast signal processing, this method allows to continuously measure the radiated magnetic field around the equipment under test. Combined with position tracking, this allows to identify areas of maximum emissions very fast. These positions are then used to obtain a more detailed spectral analysis of emissions by increasing the measurement time over those spots. In result, the whole measurement procedure can be significantly optimized by spending less time over areas of lower interest, and putting more focus on areas of potentially high emission levels. This paper presents the concept as well as an experimental comparison of three antenna movement approaches satisfying the NRE01 and RE101 radiated emissions measurement procedures.
\end{abstract}

Keywords - antenna tracking, digitiser, EMI test, fast magnetic emission test, time domain.

\section{INTRODUCTION}

An important part of electromagnetic compatibility (EMC) research is the measurement and prediction of the electromagnetic interference (EMI) emission produced by the equipment under test (EUT). One of the main challenges is the time it takes to measure the emissions around an EUT for frequencies below $150 \mathrm{kHz}$, according to for instance, test method NRE01 [1] or RE101 [2]. This measurement method use the frequency domain (FD), in which each frequency is measured and analysed separately, with a small bandwidth. For the lowest range a bandwidth of $10 \mathrm{~Hz}$ is used, which means a minimum dwell time per frequency step of $100 \mathrm{~ms}$.

Because the magnetic loop antenna is small, the whole surface of an EUT has to be scanned. The required Time to EUT can increase to several days, a weeks because of the necessity

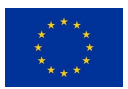

ETOPIA project research is funded by the European Union's Horizon 2020 research and innovation programme under the Marie Sklodowska Curie grant agreement No 812753 to measure in many spatial positions around it. Furthermore, an underestimation of the greatest emissions can occur in case of time-variant emission [3].

Therefore, if an EUT operates e.g. in a pulsed mode or changes its radiation characteristics over time e.g. in case of adapting to a rapidly changing load as discussed in [4], these emissions are usually hard to detect when using the conventional NRE01 measurement method. The latter underestimation can happen because the receiver switches between frequencies, measuring each frequency component separately. If the emission has a narrow frequency range and appears outside of the dwell time, it will not be detected properly. To decrease this underestimation, time domain (TD) measurements are gaining popularity [5]. According to [6], since with TD measurement all frequency components are measured at the same time, the speed of measurement is significantly increased without a sacrifice in accuracy compared to FD methods [7], [8]. The advantages and disadvantages of TDEMI analysers have been discussed in [9] and [10]. For the NRE01 standard, where the measurement frequencies are between $30 \mathrm{~Hz}$ and $100 \mathrm{kHz}$, an expensive TDEMI analyser with a mixer stage is not needed. In this frequency range, it is sufficient to use a low-cost multichannel digitizer such as a PicoScope ${ }^{\mathrm{TM}}$, which has a bandwidth of tens of $\mathrm{MHz}$ [11]. As indicated in [12], the time spent on a standard measurement of one plane of a certain large EUT is about an hour, while the time spent on a measurement using TD is reduced to a few minutes.

Continuous measurements using the standard FD method are not possible because the receiver needs to step through all the frequencies. In this case, the continuous movement of the antenna is impossible. However, TD measurements allow to move the antenna continuously because the receiver streams the broadband signal. Moving the antenna in a steady manner with constant velocity is a decent solution covering the whole space and satisfying the standard requirements for the dwell time, therefore it serves as a reference point for the work presented here. The first potential improvement suggests scanning the EUT in a sniffing manner, i.e. not following a fixed track but searching for high emission points allowing an experienced EMC test engineer to focus on these by extending the dwell time over them. However, a risk of missing some areas, as well as the underestimation of the required dwell time due to performing quick turns exists. To combat these latter issues and 
further increase the measurement effectiveness and efficiency, a technique incorporating antenna tracking is suggested in this paper. The advantage of tracking the position of the antenna is that it is possible to quickly determine the positions in which the emissions are expected to be the greatest by generating a intensity map. Such an intensity map additionally helps the test engineer to determine the spatial trends of emissions. This allows them to quickly scan over the points with the lowest suspicion of strong emissions, and yet not missing any of them. On the other hand, they can pay more attention to the points with high emission potential, which then require a longer measurement time to maximize the measurement accuracy of the emission peaks. Furthermore, due to the tracking, the results from all the measurement points are stored and add up to the total dwell time in those points. This means that every part of the measurement satisfies the NRE01 standard, as opposed to the classical sniffing approach, which serves actually as a prescan tool.

This paper discusses a measurement technique proposal that allows to:

- link the real time received TD data with the position of the antenna;

- continuously calculate the frequency spectrum using STFFT;

- ensure that no area is bypassed and the whole EUT surface is covered;

- place more focus on the suspicious areas by dynamically increasing the dwell time over them;

- obtain an intensity map of the measured surface with information about the suspected areas with potentially high emissions approaching or exceeding the standard limit line;

\section{MEASUREMENT SETUP}

The setup presented in this section is based on the TD measurement technique described in [6]. The significant improvements with respect to the standard FD measurements create a strong base to the further improvements discussed in Section III. In this work, a $13.3 \mathrm{~cm}$ loop antenna was used according to the NRE01 standard for measurements in the frequency range between $30 \mathrm{~Hz}$ and $100 \mathrm{kHz}$. Fig. 1 shows the EUT in the form of a cabinet. Inside the cabinet, there was an arbitrary signal generator with another loop antenna acting as a strongly radiating element within the EUT. The signal generator was delivering a $50 \mathrm{kHz}$ square wave amplitudemodulated signal with a $1 \mathrm{~Hz}$ sine with $100 \%$ modulation depth to emphasise the effect of time variant emissions. It is important to note that this EUT serves merely as an example to support the concept of the presented measurement technique.

Fig. 2 describes the measurement equipment, which was used in the proposed method. The PicoScope ${ }^{\mathrm{TM}} 4824$, which has $20 \mathrm{MHz}$ bandwidth, $80 \mathrm{MS} / \mathrm{s}$ [13], was used as a receiver to digitize the analogue signal received from the antenna. All of the digital signal processing was performed on a computer. The TD data was processed using short-time fast Fourier transform (STFFT) in MATLAB. Two types of detectors, peak and average, were used in accordance to [14] to generate realtime FD plots that could then be compared with the standard limit lines.

The novelty suggested in this paper is the principle of applying antenna tracking. Here, the tracking of the antenna is carried out by processing a video signal received by a camera, shown in Fig. 2, in MATLAB. This method is suggested due to the fact that it does not require special equipment. Furthermore, video tracking is attractive because it allows to measure the position of the antenna without affecting the actual emission measurement. The preferred tracking and its discussion is however outside of the scope of this paper.

\section{MEASUREMENT TECHNIQUES}

Table I shows the basic parameters for the NRE01 standard measurement. In the case presented here, the EUT is a cabinet with some electronics inside, as described in the previous section. The total scanning area is around $100 \mathrm{~cm}$ by $60 \mathrm{~cm}$. In [11], [15], the number of suggested measurement points around a large EUT, windmill motor, was demonstrated.

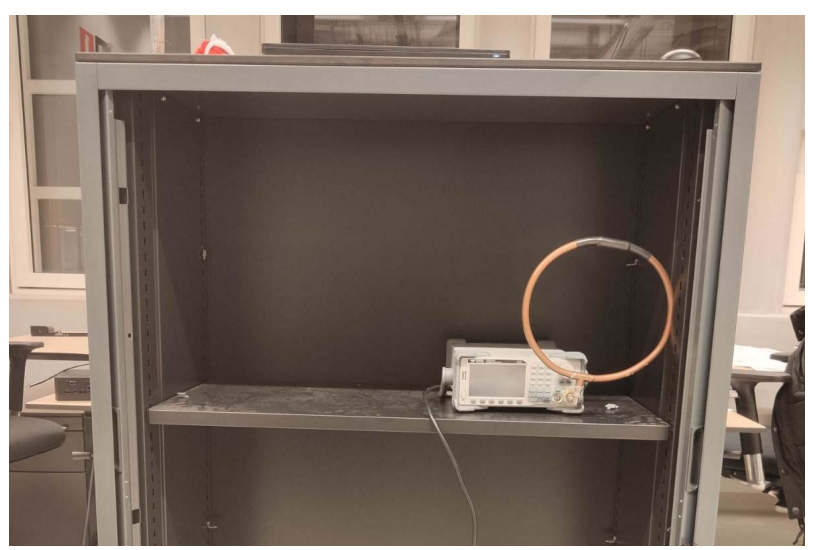

Fig. 1. An arbitrary signal generator with a loop antenna placed in a cabinet mimicking an EUT

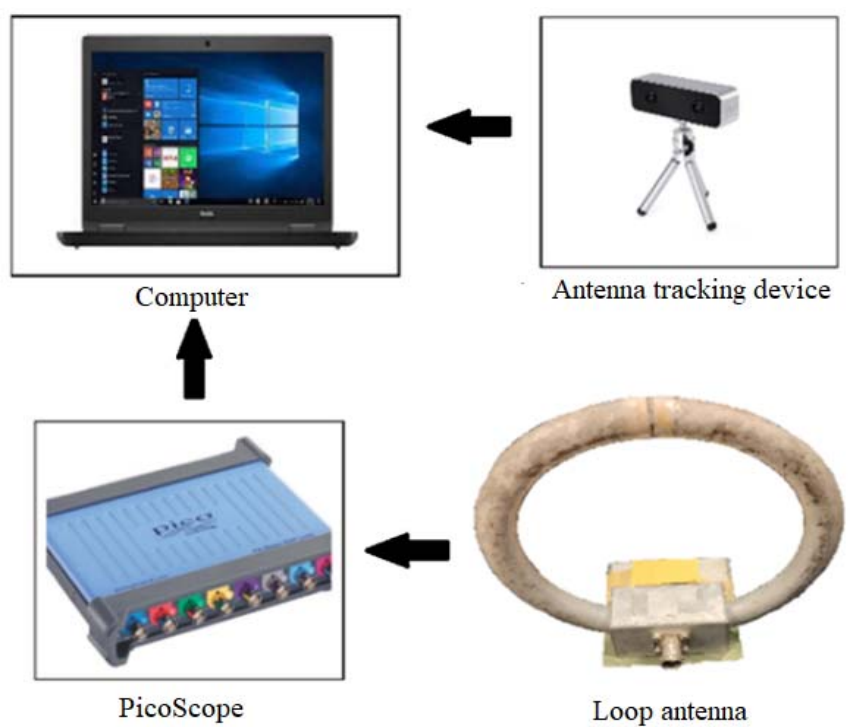

Fig. 2. Equipment used in the measurement 
Measurement positions were limited in order to shorten the long measurement time using a standard method. With the EUT presented here, 35 FD NRE01 standard measurements lasting around $67 \mathrm{~s}$ each are required for each spatial position, which in total takes around $40 \mathrm{~min}$.

TABLE I. PARAMETERS FOR STANDARD NRE01

\begin{tabular}{|l|c|c|c|c|}
\hline $\begin{array}{c}\text { Frequency } \\
\text { Range }\end{array}$ & Bandwidth & $\begin{array}{c}\text { Step } \\
\text { size }\end{array}$ & $\begin{array}{c}\text { Minimum } \\
\text { dwell time }\end{array}$ & $\begin{array}{c}\text { Minimum } \\
\text { measurement time } \\
\text { for step }\end{array}$ \\
\hline $\begin{array}{l}30 \mathrm{~Hz}- \\
1 \mathrm{kHz}\end{array}$ & $10 \mathrm{~Hz}$ & $5 \mathrm{~Hz}$ & $0.15 \mathrm{~s}$ & $0.015 \mathrm{~s}$ \\
\hline $\begin{array}{l}1 \mathrm{kHz}- \\
10 \mathrm{kHz}\end{array}$ & $100 \mathrm{~Hz}$ & $50 \mathrm{~Hz}$ & $0.015 \mathrm{~s}$ & $0.15 \mathrm{~s}$ \\
\hline $\begin{array}{l}10 \mathrm{kHz}- \\
100 \mathrm{kHz}\end{array}$ & $1 \mathrm{kHz}$ & $500 \mathrm{~Hz}$ & $0.015 \mathrm{~s}$ & $0.015 \mathrm{~s}$ \\
\hline
\end{tabular}

\section{A. Uniform motion method}

Using the TD technique described in [6] allows to measure the whole spectrum within a single dwell time provided by Table I. To obtain a measurement result that will be close to the FD measurement results, the antenna movement speed must be at most

$$
V_{\text {antenna }}=\frac{d_{\text {antenna }}}{T_{\text {point }}}
$$

where $d_{\text {antenna }}$ is the diameter of the antenna, and $T_{\text {point }}$ is the time needed for the antenna to be above the given point to obtain accurate emission data. Otherwise, if the interval of occurrence of emission with a high level is greater than the interval the antenna is located above the measurable region, peaks will not be detected. In the case of $T_{\text {point }}=0.15 \mathrm{~s}$, the total measurement takes at least $5 \mathrm{~s}$, which is already a significant improvement. However, if the peak interference appears every $1 \mathrm{~s}$ due to the time variant nature of the emission, $T_{\text {point }} \geq 1 \mathrm{~s}$ would have to be applied to every spatial position to ensure proper capturing that peak. In this case, the time to scan the whole area is at least $32 \mathrm{~s}$. This originates from the fact that the time spent at every measurement point is the same. This means that time spent for points with high and low emission levels are the same, which can make the whole measurement unnecessarily time-consuming. It is worth noting that the mentioned here values work with a perfectly uniform motion, which is difficult to achieve in a manual measurement. Unless a dedicated automated positioning system is used, the values given here are the hypothetical minimum measurement time, and are usually larger in the actual measurement.

\section{B. Sniffing method}

A step to improve the aforementioned TD continuous measurement method is to optimize the antenna movement as well as speed depending on the behaviour of the measured emissions. Generally, large electronic equipment does not radiate uniform from every point, therefore it is necessary to perform the measurement over its whole surface. It is also necessary to measure the maximum emissions, whereas the areas of low emissions are of low interest. The suggested optimization lies in quickly scanning over points of low interest, and stay longer at suspicious areas that could give higher emission levels, directly affecting the test results. Moving the receiving antenna in a manner similar to classical sniffing [16] allows the EMC test engineer to choose which positions they intend to focus on, and which to neglect. The dwell time can thus be adapted on the fly during the measurement. There are however two major drawbacks with this method, potentially caused by human factors that persist even when a real time preview of the results is available. Firstly, the dynamically adjusted trajectory of the antenna motion can cause sudden accelerations that might in turn cause the antenna speed to exceed the maximum allowed value from(1). Although such exceptions could be allowed and even beneficial in the low emission areas, the standard requirements are no longer satisfied. The problem here lies in the fact that this issue cannot be verified as there is no control or information about the actual $T_{\text {point }}$ value. Secondly, due to the inconstency of the antenna motion, certain, even critical, areas might be missed and there is no information whether such an error occurred during the measurement or not.

\section{Sniffing method with antenna tracking}

The method of continuous electromagnetic emission measurement with antenna tracking that is proposed in this article is a combination of the two methods listed above. It removes the disadvantages that were previously mentioned in the sniffing method while maintaining its very high time efficiency capabilities, while ensuring that the measurement is performed correctly and in all positions. Using the sniffing approach to search for suspicious points, the time spent on measuring the points with a low level of interference is reduced. This can significantly decrease the total measurement time due to the increased speed of movement of the antenna over an area with a low level of interference. The requirement from (1) is however satisfied because the antenna position as a function of time, and thus the corresponding temporal $T_{\text {point }}$ values, are stored and accessible from the memory. Even in a case when the antenna moves too fast, it is possible to backtrack to a previously incompletely measured point and continue measuring over it. The measured data is then combined with the previously measured data, which effectively sums and extends the total dwell time in that point.

On the other hand, points of higher priority can be measured more accurately by purposely extending the dwell time over them. A strong advantage over the continuous measurement described in Section III A is the ability to change the direction of the antenna during the measurement, and increase or decrease the antenna speed over the EUT. As an example, if a point with a high level of emission is detected, it is possible to stop above this point for a longer while to obtain more data about the time variant emissions. After proceeding with measurements around this point the test engineer can search for trends of increasing field intensity or continue measuring in another direction.

Lastly, because the results as well as the antenna positions measured using the antenna tracking are stored, it is possible to 
generate an intensity map of the high emission areas. In this paper, it is suggested to display the difference of the measured frequency spectrum and the NRE01 limit line as described in the following section. Such a solution is recommended for the sake of clarity.

\section{EXPERIMENTAL COMPARISON OF THE METHODS}

This section provides an experimental analysis addressing the strengths and the weaknesses of the discussed methods. The measurements were performed on the same EUT described in Section II. The uniform motion method measurement utilizing a constant antenna velocity was taken in around $10 \mathrm{~s}$ to ensure satisfying the standard requirements for $T_{\text {point }}=0.15 \mathrm{~s}$. Because the measurement is performed manually, and although the average velocity can be calculated from the video tracking, the actual motion performed by the engineer is never really uniform. Therefore, it is better to make sure that this requirement is satisfied by slightly extending the measurement time. The results of both peak and average detectors are shown in Fig. 3. As can be seen, the generated $50 \mathrm{kHz}$ signal is the dominant component in the visible spectrum and almost reaches the NRE01 limit line without actually exceeding it. In this case, the EUT would pass the test for radiated emission in this frequency range.

The measurement was repeated with the same EUT using the sniffing method described in Section III B. The results shown in Fig. 4 clearly state that the $50 \mathrm{kHz}$ emission level is lower than in case of the uniform motion method. This underestimation is related to the issues addressed before. The temporal accelerations of the antenna movement combined with the possibility of missing certain areas on the surface of the EUT can lead to not being able to properly capture the highest emissions. Furthermore, the repeatability of this measurement is poor due the strong effect of human factors. Therefore, although in this case the EUT would also pass the emission test, this method is strongly discouraged.

The sniffing method becomes an entirely new approach with antenna tracking as applied according to Section III.C. The experiment was repeated once again but this time the real-time STFFT-processed streamed data from the PicoScope ${ }^{\mathrm{TM}}$ was linked with the antenna tracking software. The path of the antenna used in this test is shown in Fig. 5. The initial movement starting from the top left corner of the EUT resembles the uniform motion method. The velocity in this area satisfied the requirement for $T_{\text {point }}=0.15 \mathrm{~s}$ from Table I so the movement was rather fast. However, once the test engineer noticed an increase of the radiated emissions around the right side of the EUT, he considered it to be a suspicious area and decided that it requires extra attention. By moving the antenna in the vicinity of that point, he was able to significantly extend the local dwell time at will. The intensity map indicating the number of measurements performed in each point in space is shown in Fig. 6. It is clearly shown there that the whole EUT area is covered with at least a single measurement. The areas of increased suspicion have overlapping measurements, therefore the total dwell time in those spots is summed, reaching up to over $2 \mathrm{~s}$. Such an increased dwell time allowed to observe the time variant behaviour of the source and thus properly capture the peak emission. The peak value measured at $50 \mathrm{kHz}$ shown in Fig. 7 is therefore higher than in the previous two cases and exceeds the limit line. The sampled TD data is continuously streamed and processed in real time to generate a FD result for each measured position. Each FD result is compared with the NRE01 limit line. The lowest value of the difference between them measured over the combined dwell time, i.e. the highest emission approaching or exceeding the limit line for any frequency is stored in memory, linked to the spatial position where it was measured, and displayed in the form of an intensity map as shown in Fig. 8. Such a map becomes a very useful tool in understanding the behaviour of the radiation and finding trends of emissions that then allow to track down the suspicious areas with more ease.

Of course, increasing the dwell time over the suspicious areas increases the total measurement time. In this case, the whole measurement took $40 \mathrm{~s}$, although it could have been done even faster. That is a significant increase with respect to the $10 \mathrm{~s}$ measurement time used in the uniform motion method, but the extra time was used efficiently and effectively. Only areas where it was necessary to increase the time were affected, and

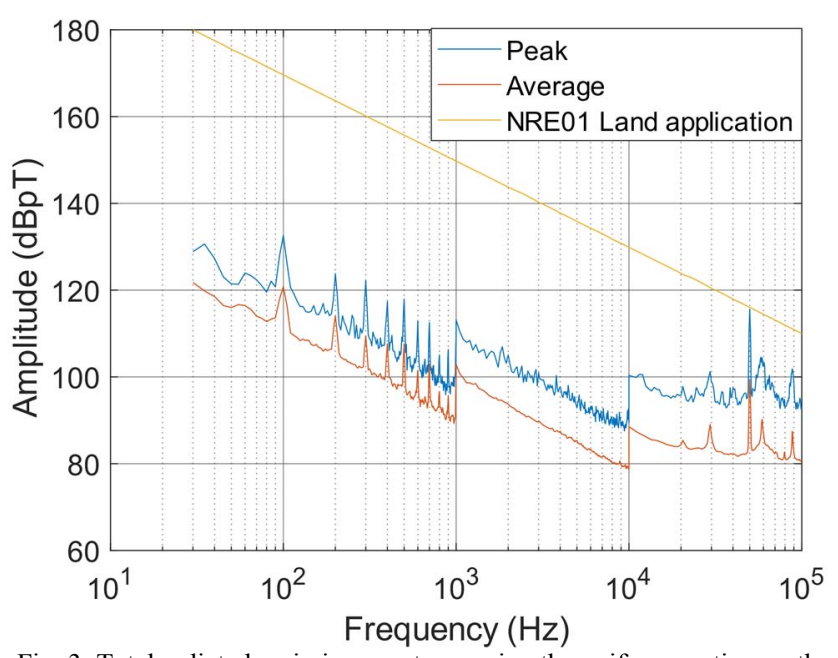

Fig. 3. Total radiated emission spectrum using the uniform motion method

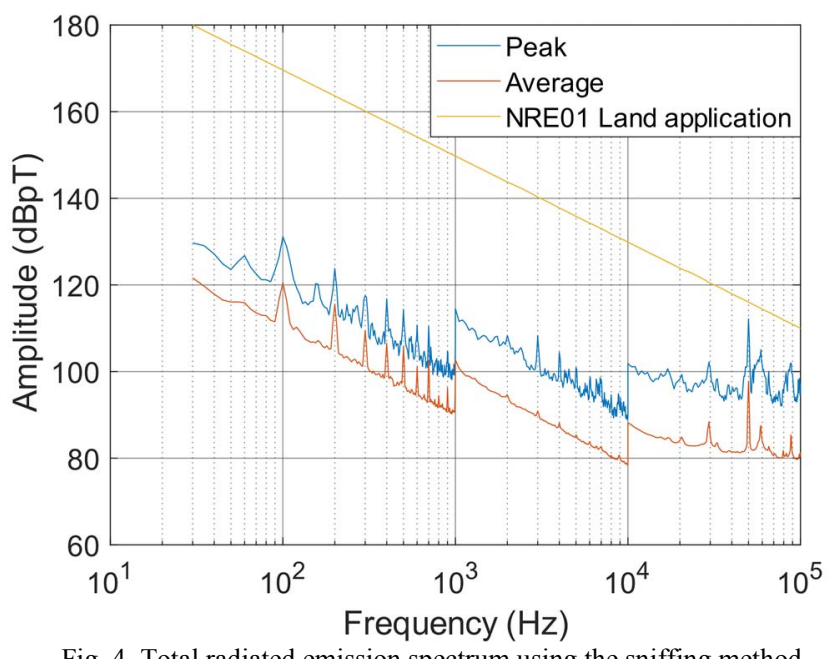

Fig. 4. Total radiated emission spectrum using the sniffing method 
this allowed to capture the real emission peaks that were missed using the previous two methods. Based on [8] it can be stated that the accuracy of the proposed TD measurement method with respect to the traditional FD one is almost the same. Also, [5] reports an advantage of performing a continuous measurement over a stationary one.

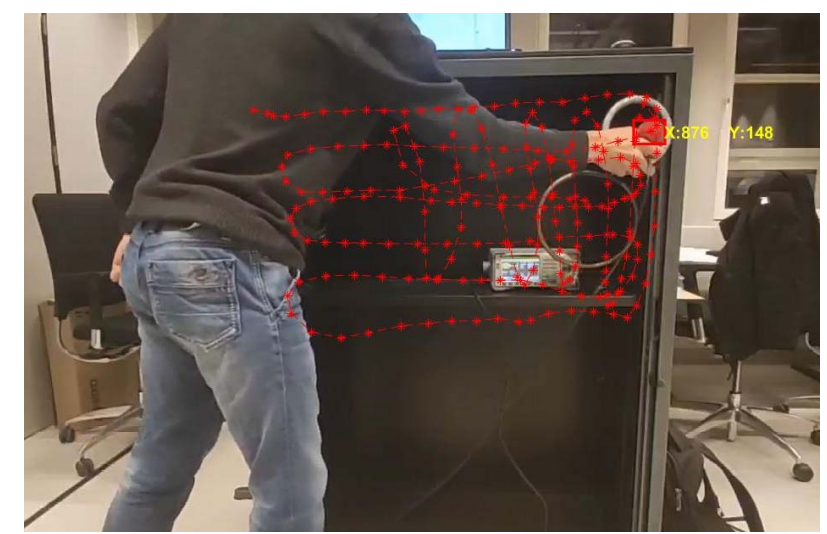

Fig. 5. The measurement path over the EUT recorded using the video antenna tracking for the sniffing method

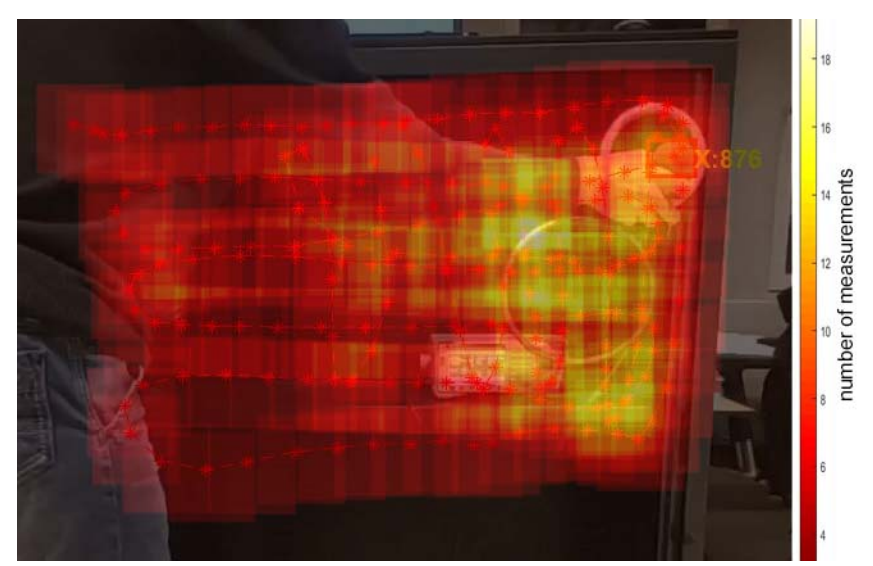

Fig. 6. Intensity map indicating the number of measuremets performed in each point in space

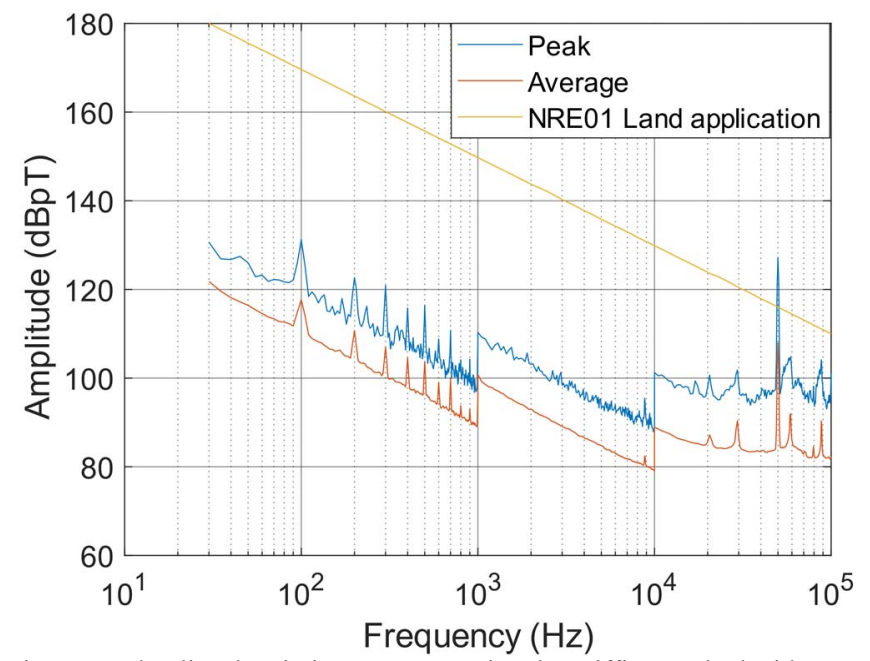

Fig. 7. Total radiated emission spectrum using the sniffing method with antena tracking

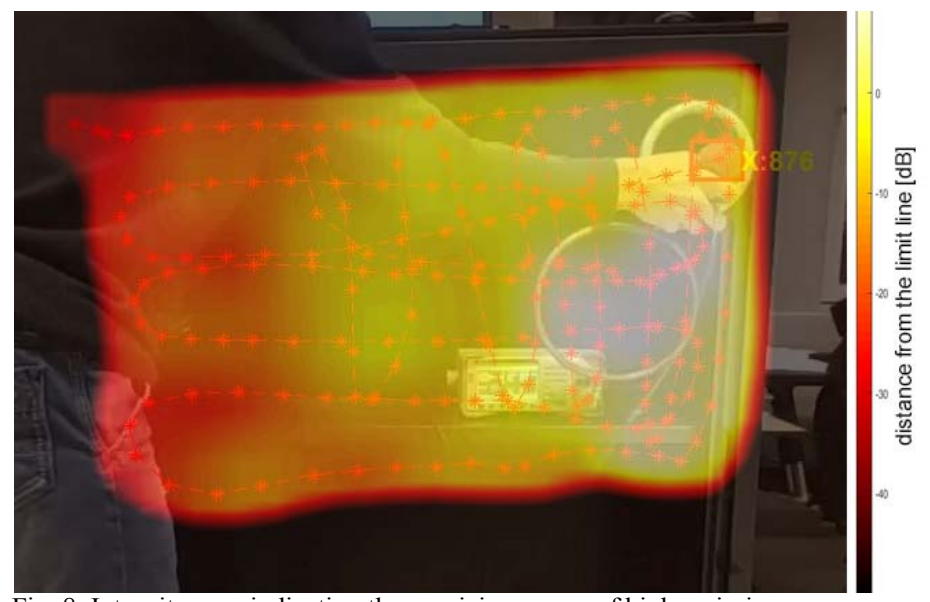

Fig. 8. Intensity map indicating the suspicious areas of high emissions

\section{CONCLUSION}

The advantages of using a cost-efficient measurement setup for performing radiated magnetic emission tests according to the NRE01 standard have been discussed. Although the application of TD measurement techniques is a significant improvement over the very time-consuming FD standard method, further improvements have been suggested. An experimental analysis of three antenna movement methods discussed in this paper have been performed. The antenna movement methods consist of a uniform motion, sniffing method and sniffing method in conjunction with antenna tracking. The uniform motion method is the most suitable solution for satisfying the NRE01 standard requirements when the time efficiency is important. It eliminates the effect of human factors as well as benefits from high repeatability of the positions of the antenna, as it always follows the same path with constant velocity, thus granting similar dwell times over each measurement point. The sniffing method has been shown to provide the least reliable results because the effect of the human factors is very high and some measurement points can be missed. However, the idea of including the antenna tracking to the sniffing method dramatically changes the performance of the latter technique. The issue of accidentally bypassing measurement points is entirely eliminated. Furthermore, this method gives the EMC test engineer the possibility to dynamically extend the measurement time over areas with a suspicion of high emissions. This advantage is crucial in cases where the behaviour of the radiated signal varies over time like in cases of emissions from power supplies adapting to changing loading conditions.

\section{REFERENCES}

[1] Electromagnetic environmental effects tests and verification, NATO standard STANAG 4370 - AECTP 501, edition E, version 1, Jan 2016.

[2] Requirements for the control of electromagnetic interference characteristics of subsystems and equipment, Department of defence interface standard MIL-STD 461G, version G, 11-Dec-2015.

[3] M. Pous and F. Silva, "Full-spectrum APD measurement of transient interferences in time domain," IEEE Trans. Electromagn. Compat., vol. 56, no. 6, pp. 1352-1360, Sep. 2014. 
[4] I. Setiawan, C. Keyer, F. Buesink, and F. Leferink, "Time-frequency diversity for solving the deadlock in defining interference levels in power lines," IEEE Int. Symp. Electromagn. Compat., Nov. 2016, pp. 364-369.

[5] J. Meng, X. Zhang, L. Zhang, and Z. Zhao, "Time-domain low-frequency non-periodic transient EMI measurement system," IET Sci. Meas. Technol., vol. 13, no. 5, Jul. 2019, pp. 650-655.

[6] T. Hartman, N. Moonen, B. Ten Have, and F. Leferink, "Fast magnetic emission tests for continuous measurements around an equipment under test," Proc. 2019 ESA Work. Aerosp. EMC, Aerosp. EMC 2019, no. 1, Aug. 2019.

[7] M. Pous, M. Azpurua, and F. Silva, "Benefits of full time-domain EMI measurements for large fixed installation, " IEEE Int. Symp. Electromagn. Compat., Nov. 2016, pp. 514-519.

[8] I. Setiawan, N. Moonen, F. Buesink, and F. Leferink, "Efficient magnetic field measurements," Int. Symp. Electromagn. Compat. - EMC Europe 2017, Nov. 2017, pp. 8-11.

[9] M. Monti, E. Puri, and M. Monti, "The importance of overload revealing in EMI receivers," Int. Symp. Electromagn. Compat. - EMC Europe 2017, Nov. 2017, pp. 14-19.

[10] K. Hörmaier, H. Zangl, and H. Zojer, "An EMI receiver model to evaluate electromagnetic emissions by simulation," 2012 IEEE I2MTC - Int. Instrum. Meas. Technol. Conf. Proc., Jul. 2012, pp. 2558-2562.
[11] Pico Technology Ltd, "PicoScope 4000 series datasheet," 2015, [Online]. Available: https://www.picotech.com/download/datasheets/PicoScope4000SeriesD ataSheet.pdf.

[12] SFGW/TR 9: Technical guidelines for wind turbines (FGW guideline) Part 9:2014, Rev. 1, Determination of high frequency emissions from renewable power generating units, 2014.

[13] Pico Technology Ltd, "PicoScope 4824 model datasheet," 2014, [Online]. Available: https://www.picotech.com/download/datasheets/picoscope-4824-datasheet.pdf.

[14] T. Karaca, B. Deutschmann, and G. Winkler, "EMI-receiver simulation model with quasi-peak detector," IEEE Int. Symp. Electromagn. Compat., Sep. 2015, pp. 891-896.

[15] S. Koj, A. Hoffmann, and H. Garbe, "Measurement uncertainty of radiated electromagnetic emissions in in situ tests of wind energy conversion systems," Adv. Radio Sci., vol. 16, Sep. 2018, pp. 13-22.

[16] D.C. Smith, "Signal and noise measurement techniques using magnetic field probes", 1999 IEEE International Symposium on Electromagnetic Compatability., 2-6 Aug. 1999, pp. 559-563. 